but is hindered by asymptomatic infections and analyses based on people tested for clinical reasons that could differ by age and gender. If improved serological detection of CT infection were available, epidemiological studies could more confidently estimate past exposure. We have explored CT incidence by age period in a cohort study, using a combination of a recently characterised serological assay (with higher sensitivity and high persistence) and self-reports.

Methods Sexual health and behaviour information was collected from a cohort of initially 1,037 participants born in Dunedin, New Zealand in 1972/3, at regular intervals up to age 38. Sera drawn at ages 26, 32 and 38 were tested for antibodies to CTspecific Pgp3 antigen using a double-antigen sandwich enzymelinked immunosorbent assay. CT incidence was examined by gender, age and number of partners.

Results By age 38, 31.5\% (146/464) women and 21.8\% (102/ 469) men had been seropositive and/or self-reported CT infection. More occurred before age 26 than in the 12 -year period 26-38 years, the difference being more marked in women than men. In all age periods the risk of acquiring CT increased with number of partners. Once the age-period specific incidence rates were adjusted for the number of partners there was no relationship between CT risk and age period. Overall the partner number adjusted risk was lower in men, although this may reflect that men are less likely to seroconvert than women.

Conclusions CT infection was very common amongst this cohort by age 38. Adjusted analyses showed a major risk factor was number of partners, with no interaction by age-period. The increased risk in men must be interpreted cautiously due to the known difference in serological responses between men and women.

Disclosure of interest statement This study was funded by Health Research Council of New Zealand. No pharmaceutical grants were received in the development of this study.

\section{P08.12 INSIGHTS INTO CHLAMYDIA TRACHOMATIS CUMULATIVE INCIDENCE IN THE CONTEXT OF WIDESPREAD OPPORTUNISTIC CHLAMYDIA SCREENING IN ENGLAND: SEROPREVALENCE STUDY USING SERA FROM A NATIONALLY-REPRESENTATIVE HOUSEHOLD SURVEY}

${ }^{1,2}{ }^{2} \mathrm{C}$ Woodhall ${ }^{*}{ }^{3}$ Gillian Wills, ${ }^{4} \mathrm{P}$ Horner, ${ }^{5} \mathrm{R}$ Craig, ${ }^{6} \mathrm{JS}$ Mindell, ${ }^{7} \mathrm{G}$ Murphy, ${ }^{3} \mathrm{M}$ MCClure, ${ }^{1} \mathrm{~K}$ Soldan, ${ }^{1} \mathrm{~A}$ Nardone ${ }^{\#},{ }^{2} \mathrm{AM}$ Johnson"\#. ${ }^{1}$ Centre for Infectious Disease Surveillance and Control, Public Health England, London, UK; ${ }^{2}$ Research Department of Infection and Population Health, UCL, London, UK; ${ }^{3}$ Department of Medicine, Imperial College London, London, UK; ${ }^{4}$ School of Social and Community Medicine, University of Bristol, Bristol, UK; ${ }^{5}$ NatCen Social Research, 35 Northampton Square, London, UK; ${ }^{6}$ Research Department of Epidemiology and Public Health, UCL, London, UK; ${ }^{7}$ Microbiology Services, Public Health England, London, UK; \#Joint Last Authors

\subsection{6/sextrans-2015-052270.358}

Introduction The National Chlamydia Screening Programme (NCSP) was nationally implemented in England in 2008. The programme recommends that sexually-active under- 25 year-olds are tested for chlamydia annually and on change of partner with the aim of interrupting transmission and preventing complications. We undertook a seroprevalence study to explore the impact of chlamydia screening on the cumulative incidence of infection up to 2012.

Methods Anonymised sera from participants in the Health Survey for England (HSE), a series of nationally-representative household surveys, were tested for anti-chlamydia antibodies using an ELISA based on the Chlamydia trachomatis-specific antigen Pgp3. Factors associated with seropositivity among 1644 year-olds in 2010 and 2012 (years when sexual behaviour questions were included) were investigated using logistic regression. Seroprevalence trends were investigated for 1994-2012.

Results In 2010/2012, Pgp3 seroprevalence was 24\% (95\% CI: $22 \%-27 \%)$ in women and $14 \%(12 \%-16 \%)$ in men. Seroprevalence increased with age to $34 \%(28 \%-40 \%)$ in $30-34$ year-old women and $20 \%(15 \%-27 \%)$ in 35-39 year-old men, and with numbers of lifetime sexual partners (17\% with 1-4 partners versus $43 \%$ in those with $\geq 10$ in women; $6 \%$ vs. $27 \%$ in men). $78 \%$ of seropositive 16-24 year-old women did not report a previous chlamydia diagnosis. Among 16-24 year-old women, there was no significant trend in seroprevalence over time and no difference in age-specific seroprevalence between birth cohorts exposed to different levels of chlamydia screening.

Conclusion In 2010-12, at least one third of women had been exposed to chlamydia by age $30-34$. Most of those with evidence of previous infection did not report a previous diagnosis, presenting consequent risks of transmission and complications. A decrease in cumulative incidence among young adults following the implementation of the NCSP has not yet been demonstrated. Additional years of screening may be needed to have a measurable effect on cumulative incidence. Continued monitoring of seroprevalence is required.

Disclosure of interest statement The study was funded by the Health Protection Agency (now part of Public Health England). No pharmaceutical or diagnostic company grants were received in the development of this study.

\section{P08.13 WHAT CAN PROBABILITY SURVEYS TELL US ABOUT CHANGES IN CHLAMYDIA PREVALENCE IN BRITAIN? EVIDENCE FROM THE NATIONAL SURVEYS OF SEXUAL ATTITUDES AND LIFESTYLES (NATSAL)}

${ }^{1,2} \mathrm{SC}$ Woodhall ${ }^{*},{ }^{2} \mathrm{~K}$ Soldan, ${ }^{1} \mathrm{P}$ Sonnenberg, ${ }^{1} \mathrm{CH}$ Mercer, ${ }^{1,3} \mathrm{~S}$ Clifton, ${ }^{1} \mathrm{P}$ Saunders, ${ }^{2} \mathrm{~F}$ Coelho da Silva, ${ }^{2} \mathrm{~S}$ Alexander, ${ }^{1} \mathrm{C}$ Tanton, ${ }^{1} \mathrm{~N}$ Field, ${ }^{1} \mathrm{~A}$ Copas, ${ }^{2} \mathrm{CA}$ Ison, ${ }^{1} \mathrm{AM}$ Johnson. ${ }^{1}$ University College London; ${ }^{2}$ Public Health UK; ${ }^{3}$ NatCen Social Research

\subsection{6/sextrans-2015-052270.359}

Introduction The National Surveys of Sexual Attitudes and Lifestyles (Natsal) estimated the prevalence of Chlamydia trachomatis (chlamydia) among the sexually-experienced British population in 1999-2001 (Natsal-2) and 2010-12 (Natsal-3). Chlamydia testing among young adults increased substantially between these years, partly due to the introduction of the English National Chlamydia Screening Programme, which achieved national implementation in 2008. We explored what these data might tell us about changing chlamydia prevalence among young adults over the last decade.

Methods We compared estimated chlamydia prevalence among sexually-experienced 18-24 year old men and women between Natsal-2 $(\mathrm{n}=680)$ and Natsal-3 $(\mathrm{n}=1,511)$. We carried out a sensitivity analysis which accounted for differences in the accuracy of the urine collection procedure and assay used in Natsal-2 (standard universal tube for urine collection; ligase chain reaction) and Natsal-3 (FirstBurst urine collection device; AptimaCombo2).

Results There was no significant difference between the chlamydia prevalence estimates for 18 to 24 year olds in Natsal-3 vs Natsal-2: for men, $2.6 \%$ (95\% CI: $1.7 \%-4.0 \%)$ vs $2.9 \%(1.3 \%-$ 6.3\%); OR: $0.91(0.36-2.27)$; for women, $3.2 \%(2.2 \%-4.6 \%)$ vs $3.1 \%(1.8 \%-5.2 \%)$; OR $1.04(0.53-2.01)$. The test-adjusted 